The diagnostic equivalent of a mean RHC PAP of $25 \mathrm{mmHG}$ was for a PASP $35.9 \mathrm{mmHG}$ and a MPAP of $23.3 \mathrm{mmHg}$. Pulmonary artery systolic pressure being the most often reported in general echocardiographic reports in this institution.

Conclusion These results demonstrate that TTE derived measures of Rap correlate poorly with invasive measurements. However, this has no impact on the traditional calculations for mean PAP and PASP by echocardiography, which correlate well with the RHC data.

\section{LEFT VENTRICULAR MORPHOLOGY IN ELITE ATHLETES WITH EXTREME ANTHROPOMETRY}

Gherardo Finocchiaro*, Harshil Dhutia, Michael Papadakis, Aneil Malhotra, Stathis Papatheodorou, Bode Ensam, Elijah Behr, Rajan Sharma, Maite Tome, Sanjay Sharma. St George's University of London Hospital

\subsection{6/heartjnl-2017-311726.120}

Background Body size measurements are critical in the correct assessment of cardiac adaptation to exercise in athletes. However, the impact of high body mass index (BMI) on cardiac dimensions in athletes is largely unknown. The aim of the study was to describe the normal cardiac parameters of a cohort of elite athletes characterised by BMI in the obesity range.

Methods Between 2007 and 2014, 1857 elite athletes with complete anthropometric data (age $21 \pm 5$ years, males $70 \%$ ) free from any cardiac disease after a normal echocardiogram were studied. The analysis was focused on the echocardiograms of 50 athletes (72\% rugby players) with BMI 30 and height $<1.95 \mathrm{~m}$ (Group 1). We compared them with athletes matched for age and body surface area (BSA) with height $>1.95 \mathrm{~m}$ and $\mathrm{BMI}<30$ (Group 2, $\mathrm{n}=87$ ) and age matched athletes with height $<1.90 \mathrm{~m}$ and BMI between 20 and 29 (Group 3, n=243).

Results The number of hours per week of exercise was lower in athletes of Group $1(17 \pm 6$ vs $22 \pm 7$ in Group 2 and 19 \pm 7 in Group 3, p $<0.001$ between Group 1 and Group 2). Athletes belonging to Group 1 exhibited larger left ventricular end-diastolic diameter (LVEDD) compared with Group 3 (57 \pm 6 vs $53 \pm 6 \mathrm{~mm}, \mathrm{p}<0.001)$, but not with Group $2(57 \pm 4$, $\mathrm{p}=0.98)$. Twenty-five $(50 \%)$ athletes of Group 1 vs $33(38 \%)$ of Group 2 and $31(13 \%)$ of Group 3 had a LV end-diastolic diameter $>57 \mathrm{~mm}(\mathrm{p}<0.001$ between Group 1 and Group 3, $\mathrm{p}=0.23$ between Group 1 and Group 2). Left ventricular (LV) wall thickness was higher in athletes of Group 1 (11 \pm 1 vs 10 \pm 2 in Group 2, $p=0.001$, vs $9 \pm 1$ in Group 3, $p<0.001)$. Twelve (24\%) athletes in Group 1 vs $19(21 \%)$ in Group 2 and $16(6 \%)$ in Group 3 exhibited a LV wall thickness $>11$ $\mathrm{mm} \quad(\mathrm{p}<0.001$ between Group 1 and Group 3, $\mathrm{p}=0.85$ between Group 1 and 2). Left atrial diameter was significantly higher in Group 1 compared to Group $3 \quad(40 \pm 5$ vs 36 $\pm 1 \mathrm{~mm}, \mathrm{p}<0.001)$. BMI had a lower correlation coefficient for LVEDD with respect to BSA $(r=0.39, p<0.001$ vs $r=0.59$, $\mathrm{p}<0.001)$.

Conclusions Athletes with BMI 30 are characterised by significantly increased LV size and left ventricular hypertrophy with wall thickness exceeding normal values in one in four cases.

\section{MID-TERM ECHOCARDIOGRAPHIC FOLLOW UP OF TRANS-CATHETER AORTIC VALVE IMPLANTATION AND INCIDENCE OF VALVULAR DEGENERATION}

Daniel Bowen, Ugochukwu Ihekwaba*, Shaun Robinson, Karen Parker, James Boyd, Cameron Densem, Bushra Rana. Papworth Hospital

\subsection{6/heartjnl-2017-311726.121}

Purpose Trans-catheter Aortic Valve Implantation (TAVI) is a well-established procedure in severe aortic stenosis with high surgical risk. However an understanding of the longer-term haemodynamic flow profile of these valves is limited, as assessed by echo parameters. This study aims to collate several key echocardiographic parameters over mid term follow up and highlight incidence rates of valvular degeneration.

Methods 49 consecutive post TAVI patients seen within the Papworth physiologist-led valve service underwent retrospective analysis performed using standard 2D/Doppler-derived echo data. Data was compared during three follow up intervals (FU1 - 3 months; FU2 - 1 year; FU3 - 2 years) and between different TAVI sizes (23, 26 and $29 \mathrm{~mm}$ ).

Results Data was analysed in all 49 patients, 26 patients reached FU3 (53\%), with a total mortality rate of $8 \%$. The majority had Sapien XT valves implanted (Sapien - 8; SapienXT - 34; Sapien3 - 7). Mean prosthetic valve Doppler measurements were similar from FU1 to FU3 - MPG $(11.5$ $\pm 7.2 ; 12.1 \pm 7.3 ; 13.3 \pm 9$ FU1-3 respectively), AVA $(1.70$ $\pm 0.59 ; 1.61 \pm 0.50 ; 1.69 \pm 0.51) \quad(\mathrm{p}=>0.05)$, whilst LVEF showed significance between FU2-FU3 (51\%-54\%; p=0.04). Secondary analysis comparing different valve sizes indicated that the smaller valve size $(23 \mathrm{~mm})$ had slightly higher peak/ mean valve gradients and lower aortic valve area (AVA), AVA index and Dimensionless Index. At FU1, the incidence of paravalvular leak (PVL) was 53\%; although significant PVL (moderate) was less than $16 \%$. Progression of PVL was noted from FU1 to FU3 only in 3\%. Within valve sizes, $29 \mathrm{~mm}$ valves

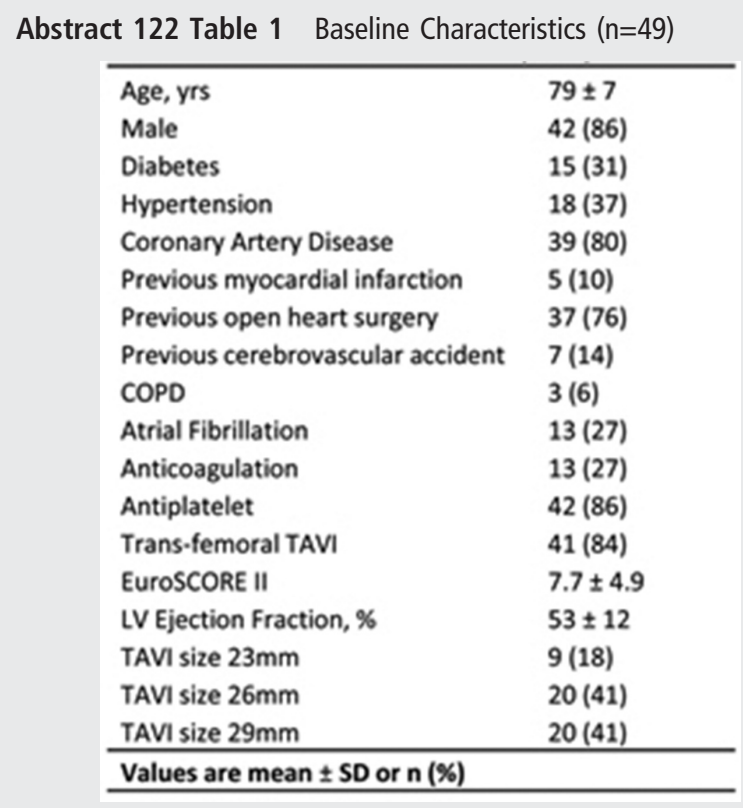

